Braz J Med Biol Res, September 2012, Volume 45(9) 862-868

doi: 10.1590/S0100-879X2012007500109

Group cognitive behavior therapy for bipolar disorder can improve the quality of life

R.T. Costa, E. Cheniaux, B.P. Rangé, M. Versiani and A.E. Nardi

The Brazilian Journal of Medical and Biological Research is partially financed by

\section{Q}

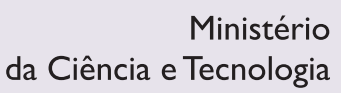

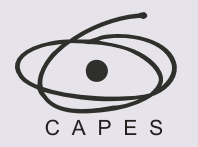

Ministério da Educação
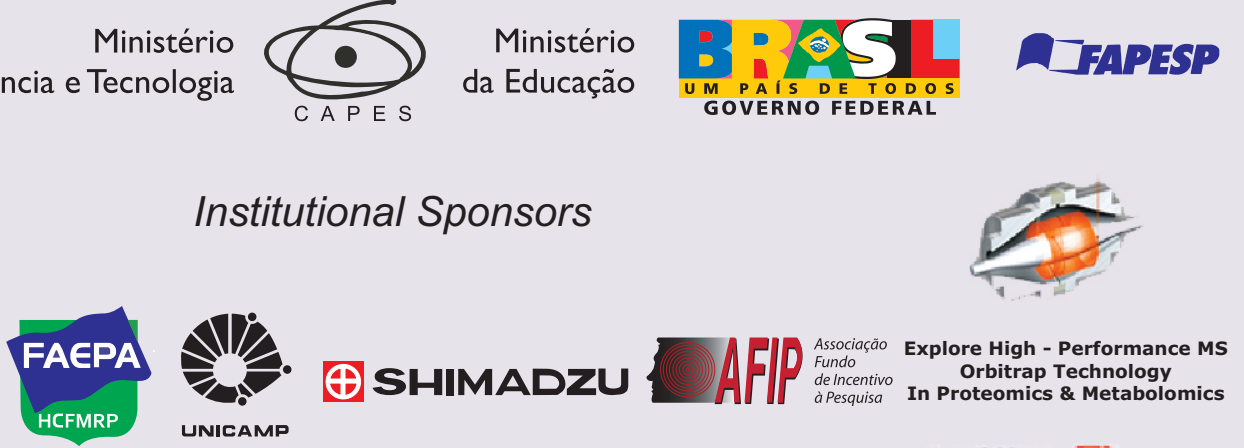

Institutional Sponsors

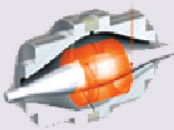

$\oplus$ SHIMADZU UNICAMP

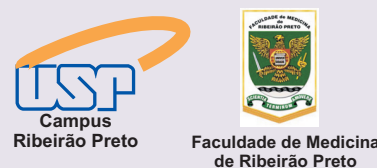
de Ribeirão Preto 


\title{
Group cognitive behavior therapy for bipolar disorder can improve the quality of life
}

\author{
R.T. Costa ${ }^{1}$, E. Cheniaux ${ }^{1}$, B.P. Rangé ${ }^{2}$, M. Versiani ${ }^{1}$ and A.E. Nardi ${ }^{1}$ \\ ${ }^{1}$ INCT Translational Medicine (CNPq) and Instituto de Psiquiatria, \\ Universidade Federal do Rio de Janeiro, Rio de Janeiro, RJ, Brasil \\ 2 Instituto de Psicologia, Universidade Federal do Rio de Janeiro, Rio de Janeiro, RJ, Brasil
}

\begin{abstract}
Bipolar disorder (BD) can have an impact on psychosocial functioning and quality of life (QoL). Several studies have shown that structured psychotherapy in conjunction with pharmacotherapy may modify the course of some disorders; however, few studies have investigated the results of group cognitive behavior therapy (G-CBT) for BD. Our objective was to evaluate the effectiveness of 14 sessions of G-CBT for BD patients, comparing this intervention plus pharmacotherapy to treatment as usual (TAU; only pharmacotherapy). Forty-one patients with BD I and II participated in this study and were randomly allocated to each group (G-CBT: $N=27$; TAU: $N=14$ ). Thirty-seven participants completed the treatment (women: $N=66.67 \%$; mean age $=41.5$ years). QoL and mood symptoms were assessed in all participants. Scores changed significantly by the end of treatment in favor of the G-CBT group. The G-CBT group presented significantly better QoL in seven of the eight sub-items assessed with the Medical Outcomes Survey SF-36 scale. At the end of treatment, the G-CBT group exhibited lower scores for mania (not statistically significant) and depression (statistically significant) as well as a reduction in the frequency and duration of mood episodes ( $P$ $<0.01)$. The group variable was significant for the reduction of depression scores over time. This clinical change may explain the improvement in six of the eight subscales of $\mathrm{QoL}(\mathrm{P}<0.05)$. The G-CBT group showed better $\mathrm{QoL}$ in absolute values in all aspects and significant improvements in nearly all subscales. These results were not observed in the TAU control group.
\end{abstract}

Key words: Group therapy; Psychotherapy; Bipolar disorder; Mania; Depression

\section{Introduction}

Several studies have shown that bipolar disorder (BD) can have a severe impact on psychosocial functioning and quality of life (QoL) (1-4). Some psychologically structured approaches favor the adhesion of BD patients to pharmacological treatment, with better results related to the reduction of depression symptoms than mania (5). There is growing interest in characterizing QoL in BD populations (6).

In a study in which QoL was assessed in 50 euthymic bipolar patients (7) using the Medical Outcomes Survey SF-36 (MOS SF-36) $(8,9)$, the scores of bipolar patients on all of the SF-36 subscales were significantly lower than those of controls (7).

Psychoeducation and cognitive behavior therapy (CBT) are the approaches most extensively studied for treating BD. Both of these treatment methods present consistent evidence of positive results (10-15). CBT strategies aim to manage and prevent cognitive, affective and behavioral symptoms associated with the depressive or mania phase with the patient's and, at times, the family's active cooperation $(16,17)$. These strategies intend to reduce negative consequences in psychosocial and interpersonal areas, thus improving the QoL of individuals with BD $(4,18)$.

Individual CBT has been extensively studied, and its effectiveness has been confirmed in controlled studies. These investigations found that CBT led to significant changes in dysfunctional thoughts and behaviors that could interfere with compliance with pharmacological treatment (19).

Only one article (20) has suggested that CBT is not likely to be an effective adjunctive therapy for the general population with recurrent, non-rapid cycling BD. Other studies using individual or group CBT (G-CBT) reported improvements in medication adherence, mood and social functioning

Correspondence: R.T. Costa, Instituto de Psiquiatria, Universidade Federal do Rio de Janeiro, INCT - Translational Medicine (CNPq), Av. Presidente Vargas, 633, sala 1718, 20071-905 Rio de Janeiro, RJ, Brasil. E-mail: faelthomaz@ig.com.br

Received August 27, 2011. Accepted June 11, 2012. Available online June 29, 2012. Published August 17, 2012. 
(21-34). Furthermore, when investigated over time, these gains were maintained at follow-up $(21-29,32)$. CBT shows efficacy in several significant phases of the disorder and can improve the QoL of BD patients by maintaining their euthymic phase or reducing the frequency and intensity of mania or depressive episodes (24-32).

Unfortunately, few studies have used G-CBT for BD patients $(22,27,31,32)$. Palmer et al. (22) used G-CBT and compared its results to those obtained before and after G-CBT plus pharmacotherapy. They evaluated the effectiveness of 17 weekly sessions in 6 BD patients on maintenance mood stabilizers. Their results showed a significant improvement in well-being in 2 patients and a trend to improved well-being in a third patient. Furthermore, all patients experienced improvement in social adjustment from the pre- to the post-test period.

In another study, Patelis-Siotis et al. (27) compared GCBT plus pharmacotherapy versus pharmacotherapy alone. They showed that the addition of G-CBT (14 sessions) to pharmacotherapy adherence in 38 BD patients improved their functioning and QoL. By the end of treatment, scores changed significantly in favor of the G-CBT group.

Costa et al. (31) compared the results of 14 sessions of G-CBT combined with pharmacotherapy to those obtained with the use of pharmacotherapy alone. The study sample was small, but it was relevant to have a control group. G-CBT patients presented fewer symptoms of mania, depression and anxiety, as well as fewer and shorter mood change episodes. The authors demonstrated that G-CBT improved especially depressive symptoms.

Gomes et al. (32) evaluated only euthymic patients. Adjunctive G-CBT was used to prevent recurrence of episodes in $50 \mathrm{BD}$ patients. The results were compared with those obtained with treatment as usual (TAU) alone. The authors concluded that there was no significant difference between groups about relapse, but the median time to relapse was longer for patients treated with G-CBT compared to those receiving TAU.

Castle et al. (33) and Isasi et al. (34) used psychological intervention programs in groups of BD patients to enhance relapse prevention. Both studies showed that there were lower rates of relapses and hospitalizations in the treatment group. Isasi et al. (34) investigated patients with refractory $\mathrm{BD}$.

However, we highlight that the number of G-CBT sessions, control group, subjective measures, and follow-up differed widely among the studies. Therefore, the research results cannot be compared. Some of the limitations of these studies were recognized by the authors but could be controlled in future studies. One of these limitations is the lack of a control group to compare to the experimental group. Furthermore, changes in individual medications may have accounted for the improvements, a possibility that was not assessed in these studies.

Psychoeducation is an intervention used by cognitive be- havior therapists. Group psychoeducation has long-lasting prophylactic effects in individuals with BD, significantly reducing illness morbidity and recurrence rates $(35,36)$.

The objective of the current study was to evaluate the effectiveness of 14 sessions of G-CBT in improving the mood symptoms and the QoL of BD patients, comparing this intervention plus pharmacotherapy to pharmacotherapy alone. Our hypothesis was that G-CBT would be effective.

We chose to apply the CBT on a group basis because there are few studies on this approach in groups and we could help a larger number of individuals within a shorter period of time, with best cost-effectiveness.

\section{Patients and Methods}

\section{Inclusion and exclusion criteria}

Subjects were recruited from the Institute of Psychiatry of the Federal University of Rio de Janeiro (Anxiety and Depression Program Outpatient Clinic) and were monitored between 2009 and 2010.

Inclusion criteria: men or women aged 18 to 60 years; patients who met the criteria of the Diagnostic and Statistical Manual of Mental Disorders-IV (DSM-IV) for BD I or II and had experienced at least one episode of hypomania, mania, or depression over the previous 12 months; subjects who had taken mood-stabilizing medication for a minimum of one month before therapy.

Exclusion criteria: Beck Depression Inventory (BDI) (37) score $\geq 35$ and/or Young Mania Rating Scale (YMRS) score $\geq 20$ (38); comorbid personality disorder and/or any other axis I severe psychiatric disorder (severe impact on professional, social and personal life); patients who met criteria for substance abuse or dependence; any severe physical illness; patients who required the administration of a new mood stabilizer and/or a new antidepressant during the course of treatment.

\section{Subjects}

Forty-one patients with bipolar I ( $\cong 84 \%$ in each group) and II disorder aged 18 to 55 years participated in the present study (24 women). Patients were allocated to G-CBT plus pharmocotherapy $(\mathrm{N}=27)$ or pharmacotherapy alone (TAU; $\mathrm{N}=14$ ). The number of participants in each $\mathrm{G}-\mathrm{CBT}$ varied from 5 to 6 . Only 4 participants did not respond to all of the scales. Two subjects in the TAU control group and 1 in the G-CBT group required hospitalization during the period of the trial, and one of the G-CBT participants withdrew from the study to receive individual therapy.

All subjects had been taking mood-stabilizing medication for a minimum of one month before therapy, and none of the participants required any change in dosage and/or the administration of a new mood stabilizer and/or a new antidepressant 2 months before and during the course of treatment. In the G-CBT group, 25 participants had taken lithium carbonate, and 2 had taken carbamazepine, while in 
the TAU group 13 received lithium, and only one participant received carbamazepine.

\section{Instruments}

Patients were interviewed using the Structured Clinical Interview for DSM-IV (SCID-I and -II) (39). MOS SF-36 (8) was applied pre- and post-treatment to evaluate QoL. MOS SF-36 was designed to measure health status, broadly defined, and to examine limitations in functioning related to physical activity, social activities, function in specific areas, and general health.

Mood symptoms were also assessed in all participants with the administration of BDI (37) and YMRS (38). Weekly ratings on the $\mathrm{BDI}$ were obtained, whereas YMRS was applied three times, i.e., at the beginning, middle and end of treatment. The participants were evaluated in a blind manner in the application of YMRS.

\section{Treatment procedures}

The study was approved by the Ethics Committee and all participants gave written informed consent.

Patients were randomly allocated to G-CBT $(N=27)$ or TAU ( $N=14)$. A variable that influenced some participants who were previously allocated to the G-CBT was the availability to participate $2 \mathrm{~h}$ per week in the schedule of the group. In the end, it was difficult to expand the TAU group, with a consequent difference in the number of subjects in the two groups.

Subjects were assessed before, during, after, and up to 6 months post-treatment with respect to their depression, mania and QoL scores.

\section{Treatment as usual}

Twelve participants in the TAU group received sessions as prescribed by the psychiatrists who were trained to apply the tests. In this group, mood symptoms were assessed for 14 weeks in addition to a 6-month follow-up.

\section{G-CBT}

Twenty-seven subjects participated in G-CBT. CBT interventions for $B D$ are intended to educate the patient and his/her family regarding treatment for and common difficulties associated with the disorder; to teach a method for monitoring the occurrence and severity of mania or depressive symptoms (mood chart); to facilitate compliance with pharmacological treatment (psychoeducation and reality test of thoughts and beliefs); to provide psychological strategies, specifically cognitive-behavior abilities, to manage stress factors that can interfere with treatment or elicit episodes of mania and/or depression (control of the circadian rhythm, daily thought records, social skills training, problem solving, etc.), to reduce the trauma and the stigma associated with the diagnosis $(18,40)$.

An experienced clinical psychologist administered the protocol, which consisted of 14 G-CBT weekly sessions, 2 h each. The intervention was divided into 2 phases: the first phase consisted of three sessions during which the therapist focused on psychoeducation for the patients and their family (BD, symptoms, medications); in the second phase, patients learned CBT skills, including specific behavioral and cognitive interventions. The CBT treatment protocol employed in the present study is based on the treatment protocol described in 'Cognitive-Behavioral Therapy for Bipolar Disorder' by Basco and Rush (16).

\section{Statistical analysis}

Pearson chi-square and Fisher exact tests were performed to determine whether the TAU group and the G-CBT group differed with respect to demographic characteristics and co-morbid diagnoses. Statistical analysis of variance was used to test for intra- and inter-group differences between scores recorded pre-, during and post-treatment. The level of significance was set at $P<0.05$.

Regression analysis was applied to learn more about the relationship among several variables. The 14th week score was the dependent variable, and we selected gender, civil status, educational level, psychiatric comorbidity, group, time (week), and the scores of each week as predictor variables.

\section{Results}

\section{Demographic characteristics}

No significant differences in demographic variables were found between the TAU group and the G-CBT group at pre-test $(P<0.05)$, as shown in Table 1.

\section{Mood scales}

Clinical scores were categorized into subclinical, mild, moderate, and severe depression (BDI: 0-11/12-19/2035/36-63) (37), and subclinical, hypomania and mania (YMRS: 0-11/12-19/20-58) (38) before treatment.

There were no significant differences between groups in baseline scores for the BDI and YMRS scales. The average depression scores indicated mild depression in both groups, and the YMRS scores indicated subclinical hypomanic/manic symptoms in both groups.

Mood scale scores for the two groups at the beginning, middle and end of treatment are presented in Table 2.

Favorable results were also obtained for G-CBT by regression analysis. Considering all of the variables controlled in the study, the only one that was significant for the reduction of depression scores over time was the group variable, which was favorable to G-CBT $\left(R^{2}=0.909 / P=\right.$ 0.002).

The time variable was significant for the lowering of anxiety scores in G-CBT. The 11 th session was a key period for the reduction of anxiety $\left(R^{2}=0.904 / P=0.027\right)$.

Regression analysis showed that some variables were 
important to explain the reduction of some scores. We show here the level of significance for each scale: BDI scores: degree of freedom (d.f.) was 20 and the level of significance 0.000; YMRS scores: d.f. was 9 and the level of significance 0.151 ; SF-36 functional capacity scores: d.f. was 8 and the level of significance 0.000 ; SF-36 physical aspect scores: d.f. was 8 and the level of significance 0.053 ; SF-36 pain scores: d.f. was 8 and the level of significance 0.001 ; SF-36 general health status scores: d.f. was 8 and the level of significance 0.000 ; SF-36 vitality scores: d.f. was 8 and the level of significance 0.014 ; SF-36 social aspect scores: d.f. was 8 and the level of significance 0.040 ; SF-36 emotional aspect scores: d.f. was 8 and the level of significance 0.001 ; SF-36 mental health scores: d.f. was 8 and the level of significance 0.007 .

The regression analysis did not indicate any significant variable explaining the reduction of the mania scores $\left(R^{2}=0.355 / P=\right.$ $0.151)$. This last finding indicates that the model did not work well. The group variable (participating in the G-CBT) did not elucidate why there was a reduction of the mania scores.
Table 1. Demographic characteristics of bipolar subject groups before treatment.

\begin{tabular}{lcc}
\hline & TAU $(\mathrm{N}=12)$ & $\mathrm{G}-\mathrm{CBT}(\mathrm{N}=27)$ \\
\hline Gender (women) & 66.67 & 60.00 \\
Mean age (years $\pm \mathrm{SD})$ & $41.5 \pm 10.33$ & $39.6 \pm 11.55$ \\
Civil status & & \\
$\quad$ Single & 33.33 & 35.00 \\
Married & 25.00 & 30.00 \\
$\quad$ Divorced/separated & 41.67 & 35.00 \\
Level of education & & \\
$\quad$ Primary school & 8.33 & 5.00 \\
$\quad$ Incomplete high school or high school & 33.34 & 54.33 \\
$\quad$ Incomplete university education or university education & 45.9 & 40.67 \\
Psychiatric comorbidity axis I & 33.34 & 29.63 \\
No psychiatric comorbidity & 66.66 & 70.37 \\
\hline
\end{tabular}

Data are reported as percent of group members except age. TAU = treatment as usual (pharmacotherapy); G-CBT = group cognitive behavior therapy plus pharmacotherapy. No significant differences were detected in demographic variables between groups before treatment (Pearson chi-square test and Fisher exact test).

Table 2. Comparison of BDI and YMRS scores.

\begin{tabular}{lcccccc}
\hline & \multicolumn{2}{c}{ TAU group $(\mathrm{N}=12)$} & & \multicolumn{2}{c}{ G-CBT group $(\mathrm{N}=27)$} \\
\cline { 2 - 3 } \cline { 6 - 7 } & BDI & YMRS & & \multicolumn{2}{c}{ BDI } & YMRS \\
\hline Baseline & $11.67 \pm 8.39$ & $1.33 \pm 3.37$ & & $19.52 \pm 9.79$ & $9.68 \pm 8.03$ \\
7th week & $12.58 \pm 8.36$ & $3.17 \pm 3.16$ & & $12.00 \pm 7.90$ & $6.72 \pm 6.20$ \\
14th week & $13.92 \pm 9.17$ & $2.92 \pm 2.81$ & & $7.24 \pm 6.42^{*}$ & $2.24 \pm 3.22^{*}$ \\
\hline
\end{tabular}

Data are reported as means $\pm \mathrm{SD}$. TAU = treatment as usual (pharmacotherapy); G-CBT = group cognitive behavior therapy plus pharmacotherapy; BDI = Beck Depression Inventory; YMRS $=$ Young Mania Rating Scale. ${ }^{*} \mathrm{P}<0.01,14$ th week compared to baseline (ANOVA).

\section{QoL scores}

Evaluation of the baseline SF-36 scores for each group indicated that there was only a significant difference for the "Mental health" subscale $(P=0.0126)$, with the TAU group presenting slightly higher average scores. The other seven subscales showed no statistical differences in the first session.

After comparing the initial and final SF-36 scores in each group, no significant differences $(P>0.05)$ in the subscales were detected in the TAU group (Functional capacity; Physical aspect; Pain; General health status; Vitality; Social aspects; Emotional aspects; Mental health), whereas there was significant improvement in all of the subscales in the G-CBT group (Physical aspect: $P=0.031$; Pain: $P=0.019$; General health status: $P=0.042$; Vitality: $P=0.003$; Social aspects: $P=0.001$; Emotional aspects: $P=0.007$; Mental health: $P=0.017)$, except for Functional capacity $(P=0.071)$.
The overall results demonstrate that there was an increase in all of the subscale scores of the G-CBT, that is, there was a substantial increase in QoL, with an average increase of $17.8 \%$ in each subscale. In contrast, the results for the TAU group either remained the same or sustained a reduction at the end of 14 weeks.

The initial and final data of the subscales are shown in Table 3.

The regression analysis was once again favorable to G-CBT. In 6 of the 8 subscales, the group variable was significantly different in explaining the score increase: Functional capacity $\left(R^{2}=0.65 / P=0.007\right)$, Pain $\left(R^{2}=0.60 / P=\right.$ $0.015)$, General health status $\left(R^{2}=0.77 / P=0.002\right)$, Vitality $\left(R^{2}=0.46 / P=0.036\right)$, Social aspects $\left(R^{2}=0.41 / P=\right.$ $0.044)$, Emotional aspects $\left(R^{2}=0.56 / P=0.001\right)$, Mental health $\left(R^{2}=0.46 / P=0.081\right)$. Participation in $G-C B T$ was an important explanatory variable for the improvement of patients' QoL. 
Table 3. Effect of group cognitive behavior therapy on quality of life scores of bipolar disorder patients being treated with pharmacotherapy.

\begin{tabular}{|c|c|c|c|c|}
\hline \multirow[t]{2}{*}{ SF-36 } & \multicolumn{2}{|c|}{ TAU group $(\mathrm{N}=12)$} & \multicolumn{2}{|c|}{ G-CBT $(N=27)$} \\
\hline & Baseline & 14th week & Baseline & 14th week \\
\hline Functional capacity & $78.80 \pm 20.68$ & $70.00 \pm 25.85$ & $66.50 \pm 20.78$ & $77.20 \pm 20.11$ \\
\hline Physical aspect & $54.17 \pm 38.19$ & $37.50 \pm 39.17$ & $32.00 \pm 37.17$ & $53.00 \pm 29.16^{*}$ \\
\hline Pain & $76.67 \pm 16.28$ & $74.67 \pm 28.91$ & $66.52 \pm 20.62$ & $80.40 \pm 19.91^{*}$ \\
\hline General health status & $66.83 \pm 14.44$ & $60.25 \pm 21.06$ & $55.24 \pm 19.88$ & $67.20 \pm 20.55^{*}$ \\
\hline Vitality & $50.42 \pm 20.05$ & $48.33 \pm 22.39$ & $44.00 \pm 19.15$ & $61.00 \pm 19.63^{*}$ \\
\hline Social aspects & $66.04 \pm 23.54$ & $66.04 \pm 30.39$ & $48.50 \pm 26.10$ & $75.00 \pm 26.02^{*}$ \\
\hline Emotional aspects & $50.00 \pm 38.93$ & $30.56 \pm 41.38$ & $28.00 \pm 35.59$ & $56.00 \pm 34.32^{*}$ \\
\hline Mental health & $63.00 \pm 18.93$ & $60.00 \pm 19.15$ & $47.84 \pm 19.37$ & $61.18 \pm 18.86^{*}$ \\
\hline
\end{tabular}

Data are reported as means \pm SD in percent. TAU = treatment as usual (pharmacotherapy); G-CBT = group cognitive behavior therapy plus pharmacotherapy; SF-36 = Medical Outcomes Survey SF-36. *P $<0.05,14$ th week compared to baseline (ANOVA).

\section{Discussion}

Group therapy was well tolerated by the BD patients, as indicated by the fact that $92.59 \%$ of the patients participated in G-CBT from the beginning to the end of the study, supporting the report of Patelis-Siotis et al. (27).

The addition of G-CBT to the pharmacological treatment was effective. The patients presented less mania, depression and anxiety symptoms as well as a reduction in the frequency and duration of mood change episodes.

It is important to note that the depression scores of the G-CBT group were slightly higher than those of the control group at baseline, however, on average, both groups demonstrated mild depression. CBT sessions were important for the improvement of depression symptoms. In this way, we corroborated the findings of Scott et al. (26), Ball et al. (29), Reilly-Harrington et al. (20), Patelis-Siotis et al. (27), and Costa et al. (31) who investigated the efficacy of individual $(20,26,29)$ and group $(27,31) \mathrm{CBT}$, and also reported reduction in depression symptoms.

Although there was a reduction in the indices of mania, the model did not prove to be effective because regression analysis showed that none of the variables investigated influenced the reduction of mania scores. Reilly-Harrington et al. (20), who investigated 20 sessions of CBT for BD, also found that patients did not present a significant reduction of mania symptoms. Nevertheless, subjects in both groups presented low scores for YMRS since the first assessment, a complicating factor that can explain a non-significant reduction in the mania scores. This can be explained by the good and regular use of mood stabilizers throughout the 14 weeks.
The G-CBT group showed better QoL in absolute values and significant improvement in almost all aspects, excluding only "Functional capacity". This was not the case for the TAU patients. Furthermore, we corroborated the results of Patelis-Siostis et al. (27) since there was a reduction in YMRS scores, while there was also a significant improvement in the "Vitality" subscale.

The current study has several limitations. First, there was a difference in the number of subjects between groups. Second, patients with severe mania or depression were excluded. Third, although there were no significant differences in baseline scores, the G-CBT group had slightly higher mean raw scores for anxiety, depression and mania prior to treatment. This could favor a less apparent reduction of symptoms in the TAU group. Furthermore, there was a lack of follow-up to evaluate whether the patients sustained the gains achieved during therapy.

CBT used in conjunction with pharmacotherapy for BD patients may modify the course of the disease. We report that there are improvements in mood and QoL. G-CBT may be particularly useful in the treatment of bipolar depression.

When compared with other studies, the present investigation differed in number of treatment sessions, subjective measures, and follow-up. In the future, replication studies on CBT for BD patients should be conducted to control possible limitations and producing clearer results. Additional controlled studies using G-CBT and larger samples are needed to add evidence of effectiveness.

\section{Acknowledgments}

Research supported by CNPq (\#470382/2006-6). 


\section{References}

1. Cooke RG, Robb JC, Young LT, Joffe RT. Well-being and functioning in patients with bipolar disorder assessed using the MOS 20-ITEM short form (SF-20). J Affect Disord 1996; 39: 93-97.

2. Harrow M, Goldberg JF, Grossman LS, Meltzer HY. Outcome in manic disorders. A naturalistic follow-up study. Arch Gen Psychiatry 1990; 47: 665-671.

3. Robb JC, Cooke RG, Devins GM, Young LT, Joffe RT. Quality of life and lifestyle disruption in euthymic bipolar disorder. $J$ Psychiatr Res 1997; 31: 509-517.

4. IsHak WW, Brown K, Aye SS, Kahloon M, Mobaraki S, Hanna R. Health-related quality of life in bipolar disorder. Bipolar Disord 2012; 14: 6-18.

5. Sachs GS. Unmet clinical needs in bipolar disorder. J Clin Psychopharmacol 2003; 23: S2-S8.

6. Michalak EE, Yatham LN, Lam RW. Quality of life in bipolar disorder: a review of the literature. Health Qual Life Outcomes 2005; 3: 72.

7. Sierra P, Livianos L, Rojo L. Quality of life for patients with bipolar disorder: relationship with clinical and demographic variables. Bipolar Disord 2005; 7: 159-165.

8. Ware JE Jr, Sherbourne CD. The MOS 36-item short-form health survey (SF-36). I. Conceptual framework and item selection. Med Care 1992; 30: 473-483.

9. Ciconelli RM. Translation into Portuguese and validation of the generic questionnaire for assessing quality of life: "medical outcomes study 36 - item short - form health survey" (SF-36). [Doctoral Thesis]: Escola Paulista de Medicina, Universidade Federal de São Paulo; 1997.

10. Peet M, Harvey NS. Lithium maintenance: 1. A standard education programme for patients. Br J Psychiatry 1991; 158: 197-200.

11. Colom F, Vieta E. [Improving the outcome of bipolar disorder through non-pharmacological strategies: the role of psychoeducation]. Rev Bras Psiquiatr 2004; 26 (Suppl 3): 47-50.

12. Vieta E, Pacchiarotti I, Scott J, Sanchez-Moreno J, Di Marzo $S$, Colom F. Evidence-based research on the efficacy of psychologic interventions in bipolar disorders: a critical review. Curr Psychiatry Rep 2005; 7: 449-455.

13. Gomes BC, Lafer B. Group psychotherapy for patients with bipolar affective disorder. Rev Psiquiat Clín 2006; 34: 8489.

14. Khazaal Y, Preisig M, Zullino DF. [Psychoeducational and cognitive behavioral treatments of bipolar disorder]. Sante Ment Que 2006; 31: 125-143.

15. Schottle D, Huber CG, Bock T, Meyer TD. Psychotherapy for bipolar disorder: a review of the most recent studies. Curr Opin Psychiatry 2011; 24: 549-555.

16. Basco MR, Rush AJ. Cognitive-behavioral therapy for bipolar disorder. New York: Guilford Press; 1996.

17. Newman C. Bipolar disorder: a cognitive therapy approach. Washington: American Psychological Association; 2002.

18. Lam DH, Jones SH, Hayward P, Bright JA. Cognitive therapy for bipolar disorder: A therapist's guide to concepts, methods and practice. New York: John Wiley and Sons; 1999.

19. Scott J, Colom F, Vieta E. A meta-analysis of relapse rates with adjunctive psychological therapies compared to usual psychiatric treatment for bipolar disorders. Int J Neuropsychopharmacol 2007; 10: 123-129.
20. Reilly-Harrington NA, Deckersbach T, Knauz R, Wu Y, Tran $T$, Eidelman P, et al. Cognitive behavioral therapy for rapidcycling bipolar disorder: a pilot study. J Psychiatr Pract 2007; 13: 291-297.

21. Cochran SD. Preventing medical noncompliance in the outpatient treatment of bipolar affective disorders. $J$ Consult Clin Psychol 1984; 52: 873-878.

22. Palmer AG, Williams $H$, Gorsefield D, Adams M. CBT in a group format for bipolar affective disorder. Behav Cogn Psychother 1995; 23: 153-168.

23. Perry A, Tarrier N, Morriss R, McCarthy E, Limb K. Randomised controlled trial of efficacy of teaching patients with bipolar disorder to identify early symptoms of relapse and obtain treatment. BMJ 1999; 318: 149-153.

24. Zaretsky AE, Segal ZV, Gemar M. Cognitive therapy for bipolar depression: a pilot study. Can J Psychiatry 1999; 44: 491-494.

25. Lam DH, Bright J, Jones S, Hayward P, Schuck N, Chisholm $D$, et al. Cognitive therapy for bipolar disorder - a pilot study of relapse prevention. Cogn Ther Res 2000; 24: 503-520.

26. Scott J, Garland A, Moorhead S. A pilot study of cognitive therapy in bipolar disorders. Psychol Med 2001; 31: 459467.

27. Patelis-Siotis I, Young LT, Robb JC, Marriott M, Bieling PJ, Cox LC, et al. Group cognitive behavioral therapy for bipolar disorder: a feasibility and effectiveness study. J Affect Disord 2001; 65: 145-153.

28. Lam DH, Watkins ER, Hayward P, Bright J, Wright K, Kerr N, et al. A randomized controlled study of cognitive therapy for relapse prevention for bipolar affective disorder: outcome of the first year. Arch Gen Psychiatry 2003; 60: 145-152.

29. Ball JR, Mitchell PB, Corry JC, Skillecorn A, Smith M, Malhi GS. A randomized controlled trial of cognitive therapy for bipolar disorder: focus on long-term change. J Clin Psychiatry 2006; 67: 277-286.

30. Zaretsky A, Lancee W, Miller C, Harris A, Parikh SV. Is cognitive-behavioural therapy more effective than psychoeducation in bipolar disorder? Can J Psychiatry 2008; 53: 441-448.

31. Costa RT, Cheniaux E, Rosaes PA, Carvalho MR, Freire RC, Versiani $\mathrm{M}$, et al. The effectiveness of cognitive behavioral group therapy in treating bipolar disorder: a randomized controlled study. Rev Bras Psiquiatr 2011; 33: 144-149.

32. Gomes BC, Abreu LN, Brietzke E, Caetano SC, Kleinman A, Nery FG, et al. A randomized controlled trial of cognitive behavioral group therapy for bipolar disorder. Psychother Psychosom 2011; 80: 144-150.

33. Castle D, White C, Chamberlain J, Berk M, Berk L, Lauder $S$, et al. Group-based psychosocial intervention for bipolar disorder: randomised controlled trial. Br J Psychiatry 2010; 196: 383-388.

34. Isasi AG, Echeburua E, Liminana JM, Gonzalez-Pinto A. How effective is a psychological intervention program for patients with refractory bipolar disorder? A randomized controlled trial. J Affect Disord 2010; 126: 80-87.

35. Colom F, Vieta E, Reinares M, Martinez-Aran A, Torrent C, Goikolea JM, et al. Psychoeducation efficacy in bipolar disorders: beyond compliance enhancement. J Clin Psychiatry 2003; 64: 1101-1105. 
36. Colom F, Vieta E, Sanchez-Moreno J, Palomino-Otiniano R, Reinares M, Goikolea JM, et al. Group psychoeducation for stabilised bipolar disorders: 5-year outcome of a randomised clinical trial. Br J Psychiatry 2009; 194: 260-265.

37. Beck AT, Ward CH, Mendelson M, Mock J, Erbaugh J. An inventory for measuring depression. Arch Gen Psychiatry 1961; 4: 561-571.

38. Young RC, Biggs JT, Ziegler VE, Meyer DA. A rating scale for mania: reliability, validity and sensitivity. Br J Psychiatry 1978; 133: 429-435

39. First MB, Spitzer RL, Gibbon M, Williams JBW. SCID-I/P, Version 2.0. Biometrics Research Department. New York, New York State Psychiatric Institute; 1996.

40. Otto MW, Miklowitz DJ. The role and impact of psychotherapy in the management of bipolar disorder. CNS Spectr 2004; 9: 27-32. 